\title{
3D Wiener Filtering to Reduce Reverberations in Ultrasound Image Sequences
}

\author{
Nina Eriksson Bylund, Marcus Ressner, and Hans Knutsson \\ Dept. of Biomedical Engineering, Linköping University, SE-581 85 Linköping, Sweden \\ \{nina, marre, knutte\}@imt.liu.se, http://www.imt.liu.se/mi
}

\begin{abstract}
One of the most frequently occuring artifacts in ultrasound imaging is reverberations. These are multiple reflection echoes that result in ghost echoes in the ultrasound image.

A method for reducing these unwanted artifacts using a three-dimensional (3D) Wiener filter is presented. The Wiener filter is a global filter and produces an estimate of the uncorrupted signal by minimizing the mean square error between the estimate and the uncorrupted signal in a statistical sense.

The procedure works as follows: In a graphic interface the operator is displayed an image sequence. The operator marks two areas in one of the images; one area which contains a typical reverberation artifact, and one area free from artifact. Using these areas to produce noise and signal estimates, a Wiener filter is created and applied to the sequence.

The 3D Wiener filters display excellent selection capabilities, and the developed method significantly reduces the magnitude of the reverberation artifact in the tested sequences.
\end{abstract}

\section{Introduction}

Image artifacts in diagnostic ultrasound can occur for a number of reasons, all leading to a more complicated interpretation procedure as important structural information may be obscured. One of the most frequently occuring artifacts in diagnostic ultrasound imaging is reverberations. This type of artifact is caused by the incorrect assumption that the ultrasound pulse always travels in a straight line, to and from the reflecting interface. Multiple reflections between two closely spaced interfaces will therefore give rise to multiple copies of the anatomic interface. In the image, these will appear as ghost echoes at some integer multiple of the distance between the interfaces being imaged [7].

Another source of reverberations is that a small portion of the returning sound pulse may be reflected back into the tissues by the transducer surface itself, and generate a new echo at twice the depth. In echocardiography (imaging the heart), reverberations are also related to the fact that the operator needs to find an "acoustic window"; the ultrasound beam has to fit between the ribs and avoid the lungs. It is therefore not unusual that part of the beam is obstructed and reflected back and forth between the ribs and the transducer surface a number of times, before it propagates out into the tissue and generates an echo at the interface. This type of reverberation will give rise to so-called "ring down" effects 
where the interface is broadened as the registered echoes appear to come from regions at increasing depth.

Deeper lying strong reflectors such as the lungs (soft tissue-to-air interfaces) or the diaphragm can give rise to artifacts similar to reverberations. These types of artifacts appear when a strong echo from a deeper lying interface reaches the transducer after the next pulse has been transmitted. The reflecting interface will then be placed at a much shorter distance from the transducer as the backscattered echo is assumed to originate from the last transmitted pulse.

In a study performed to evaluate so-called wide range frequency estimation as a tool for ultrasound image segmentation [2], we discovered that the estimated spectrum variance was low at the reverberation artifact locations. A local frequency estimate may be seen as the average of the frequencies present at a given spatial position. This estimate's deviation from the instantaneous frequency may be interpreted as the instantaneous bandwidth [4]. For example, a signal with broad bandwidth would give a more uncertain local frequency estimate than a signal with a narrow bandwidth. In Fig. 1, the spectrum variance summed over all images in an in vivo ultrasound sequence is shown, and the small values at the reverberation location imply that this artifact have a relatively narrow bandwidth.

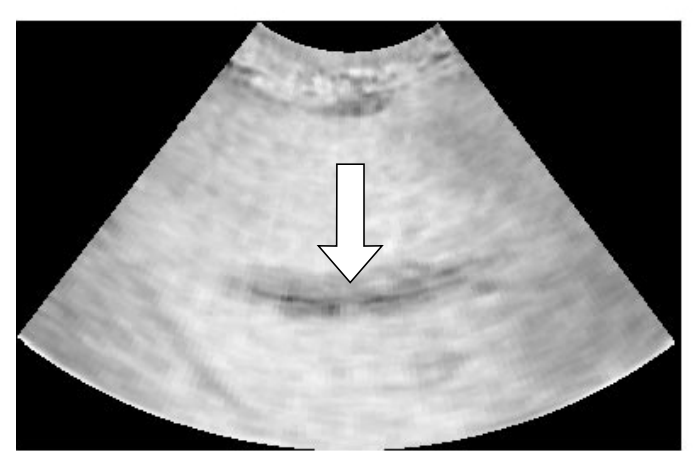

Fig. 1. The spectrum variance summed over all frames in an in vivo sequence. The reverberation artifact is pointed out.

\section{Material and methods}

In this study the Vingmed System Five was used to acquire the ultrasound images. This system performs preprocessing of the received signal in the following way [5]: the signal from each of the ultrasound probe's piezoelectric elements is amplified with a factor that varies with depth (TGC, Time Gain compensation) so that the average signal amplitude is approximately equal for all depths. The contribution from each element is delayed in order to focus the beam to a certain depth and direction. To obtain the desired apodiziation and beam profile, weights are assigned to the signals before they are summed. This sum is the real-valued, 
digital output of the ultrasound system's beamformer, and is called the Radio Frequency (RF) signal. In the Vingmed System Five, the sampling rate at the output of the beamformer is $20 \mathrm{MHz}$, and the resolution is 20 bits [5].

The Inphase Quadrature (IQ) signal is the least processed signal that is possible to retrieve from the Vingmed System Five. The IQ-data is constructed by a process called IQ-demodulation, which includes three main steps:

1. The real valued RF-signal is shifted down in the frequency domain, $f_{R F}(t) e^{i u_{d} t} \leftrightarrow F\left(u-u_{d}\right)$, where $u_{d}=2 \pi f_{d}$ is chosen so that the right part of the spectrum is centered at the origin in the Fourier domain. The shift will make the previously real signal complex, but with the same energy content.

2. The signal is low-pass filtered in order to remove the frequencies that were initially negative.

3. As the bandwidth of the signal is relatively small, sub-sampling without loss of information can now be performed.

In Fig. 2 an example of an IQ-demodulated RF-beam is shown.
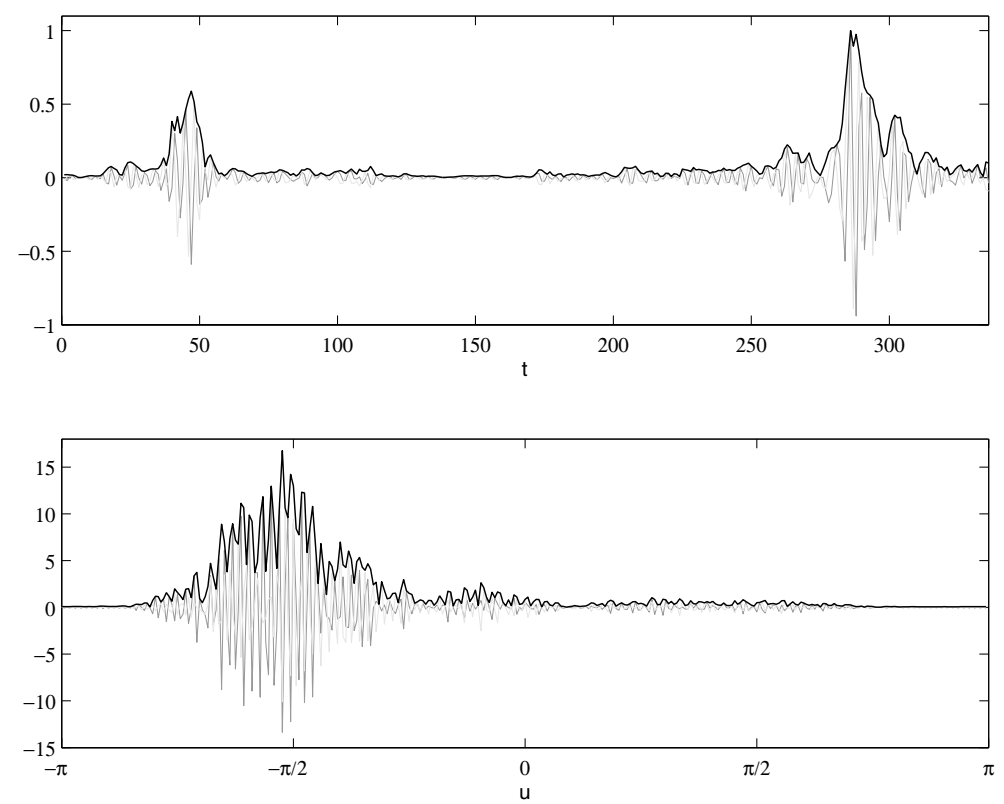

Fig. 2. The IQ-demodulated version of one beam from the beamformer output, shown in the temporal and Fourier domains. The real part of each signal is shown in a light gray shade, the imaginary part in a darker gray, and the absolute value in black.

The image shown on the ultrasound system display is saved in a format called tissue-data. In normal scanning, one tissue-image is captured between each IQ-image. Tissue-data is basically the envelope of the sub-sampled RF-signal, 
resampled to rectangular coordinates (in order to get the geometry right) and log-compressed to make the dynamic range of the data fit the human eye.

Thus, the tissue-data are amplitude images with no phase component, so that the major part of the signal energy is concentrated around the origin. In tissue harmonic imaging [1] only the harmonic components are used when creating an image from the back-scattered echoes. The reverberation noise is reduced in images acquired with this modality, indicating that the reverberation noise spectrum is also concentrated at low frequencies. Initial attempts to reduce the reverberation artifact using tissue-data proved to be unsuccesful, the signal and noise are hard to separate in images of this format. Therefore, in this study, IQ-data is used for restoration of the image sequences.

\subsection{Test sequences}

Two 2D + time ultrasound IQ-sequences were used to evaluate the algorithm: one sequence on a tissue-mimicking graphite in agar gel phantom [3], in which bars of glass simulating ribs give rise to reverberations, and one sequence on an open-chest pig with a strong reverberation from a water-filled rubber glove used as a medium between the heart and the transducer.

\section{$2.23 \mathrm{D}$ Wiener filter}

One important classical approach for attempting to improve the quality of a signal is the Wiener filter. The Wiener filter is a global filter and produces an estimate of the uncorrupted signal by minimizing the mean square error between the estimate and the uncorrupted signal in a statistical sense. The twodimensional Wiener filter is mainly used to restore images corrupted by additive noise.

In order to apply a Wiener filter, one needs knowledge about the point-spread function of the imaging device causing the addition of noise. Generally, this information is not available and a model must be used. In the case of a stationary signal, corrupted by some other additive and uncorrelated signal (referred to as the noise), the model may be stated as $\mathbf{g}=\mathbf{f}+\mathbf{n}$, where $\mathbf{g}$ is the corrupted signal, $\mathbf{f}$ is the undegraded signal and $\mathbf{n}$ is the additive noise. It can be shown (see for example [8]) that the restoration filter that finds the optimum linear estimate is given by:

$$
W(\mathbf{u})=\frac{|F(\mathbf{u})|^{2}}{|F(\mathbf{u})|^{2}+|N(\mathbf{u})|^{2}} .
$$

In this study, $|F(\mathbf{u})|^{2}$ is the three-dimensional power spectrum of the signal or undegraded image volume and $|N(\mathbf{u})|^{2}$ is the three-dimensional power spectrum of the noise, i.e. the reverberation artifact.

\subsection{Filtering process}

A scheme of the filtering process in shown i Fig. 3. In a graphic interface the operator is displayed an image sequence. The operator marks two areas in one of 


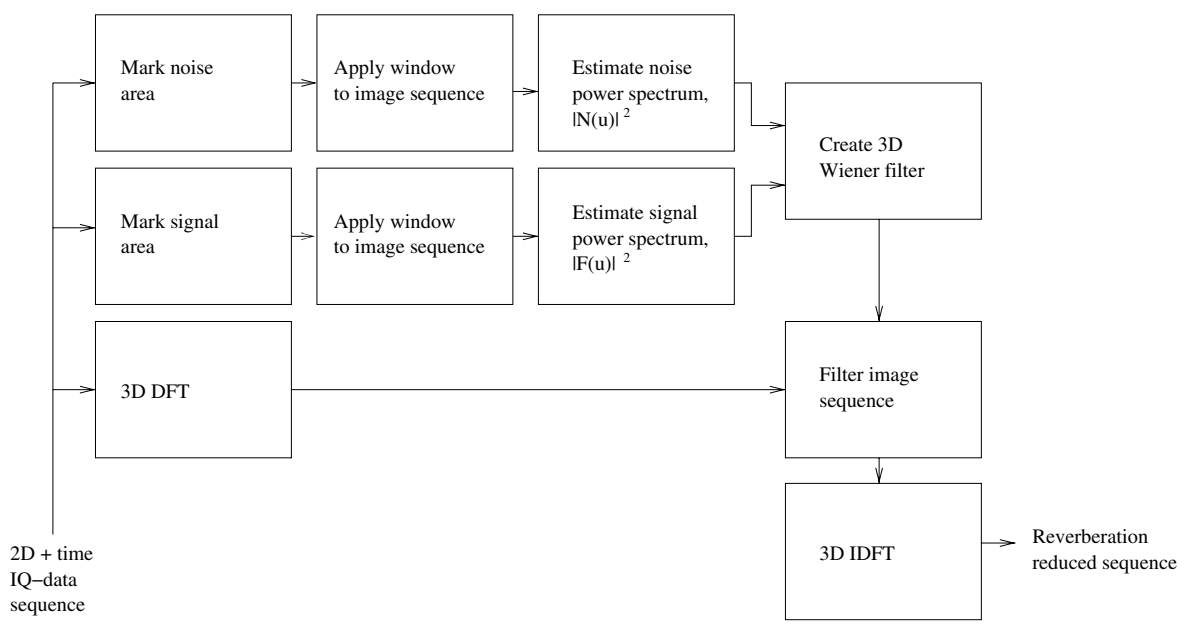

Fig. 3. A schematic illustration of the reverberation reduction algorithm.

the images; one area which contains a typical reverberation artifact, representing the noise, and one area free from artifact, representing the signal. This can be done in an image shown in so-called polar coordinates, as in the images shown in Fig. 4, or in the standard geometric coordinates as shown in Fig. 5.

The noise and signal volumes are defined by applying windows to the sequence in all three dimensions. Gaussian functions are used in order to avoid ringing effects. The width and height of the areas indicated by the operator are used as the standard deviations of the Gaussian windows for the angular and depth directions of the data. Also, in the temporal dimension the standard deviation and position of the Gaussian window may be specified.

The three-dimensional DFT for each volume is computed, and estimates of the power spectra for the signal and noise volumes are obtained by squaring the magnitude of the Fourier transformed volumes and smoothing the results with a 3D Gaussian kernel. The smoothing limits the rate of change of the power spectra, and reciprocally the required spatio-temporal size of the Wiener filter.

The Wiener filter is computed from the estimated signal and noise power spectra by the formula in Eq. 1. The image sequence is filtered by multiplication in the Fourier domain and the result is transformed to the spatio-temporal domain.

\section{Results}

The developed method significantly reduce the magnitude of the reverberation artifact in both test sequences. To demonstrate this, one image from the phantom sequence is shown in Fig. 6, and one image from the in vivo sequence is shown in Fig. 7. In both figures, the magnitude of the results from the filtering procedure is shown, resampled to geometric coordinates. 

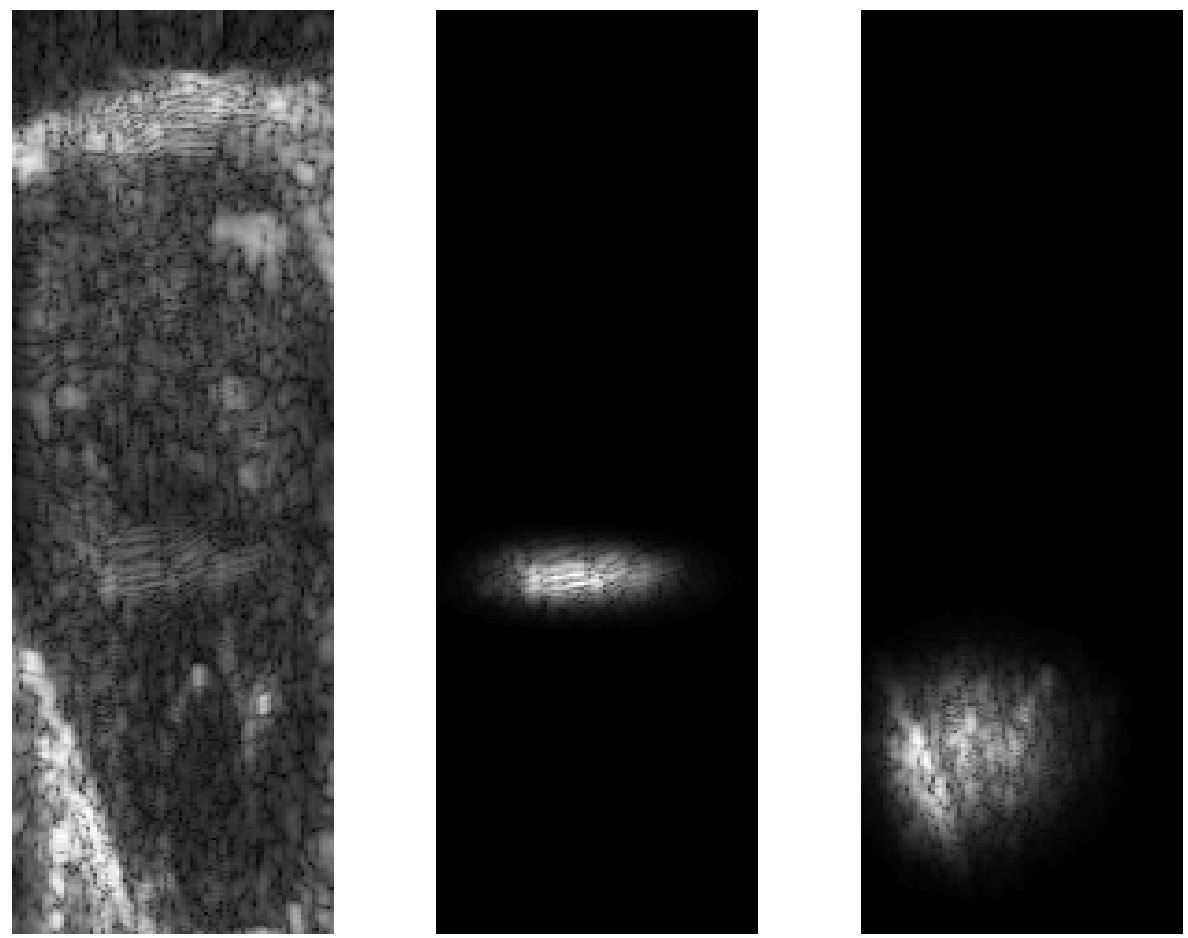

Fig. 4. One frame of the in vivo sequence, the noise area and the signal area (from left to right) in polar coordinates.

No effort has been put into producing eye-pleasing tissue sequences from the filtered IQ-data. The ultrasound system manufacturers have put a great deal of work into displaying nice images on the screen. As our intention is to produce images that are as revealing as possible we have not concentrated on mimicking what has already been done, and the images presented here can be seen as reverberation reduced data right before this last eye-pleasing processing step.

\section{Discussion}

Traditionally, processing of ultrasound data has been one dimensional. To remove the stationary reverberation originating from the ultrasound pulse bouncing back and forth between the fatty tissue interfaces under the skin and the transducer surface, Nickel [6] has proposed a high-pass filtering scheme in the temporal dimension. Each pixel of an echocardiographic RF-sequence is processed from frame to frame in real time. With this approach there is a risk of removing stationary structures in the sequence. Also, the respiration and involuntary movements of the transducer may make the reverberation less stationary. 

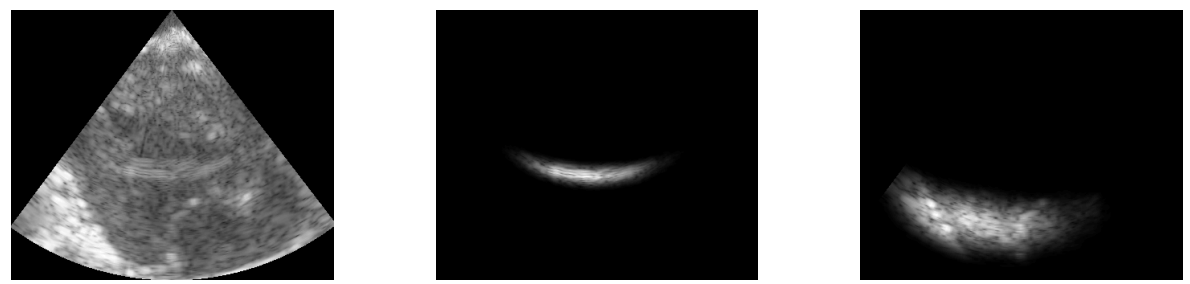

Fig. 5. One frame of the in vivo sequence, the noise area and the signal area (from left to right) in geometric coordinates.
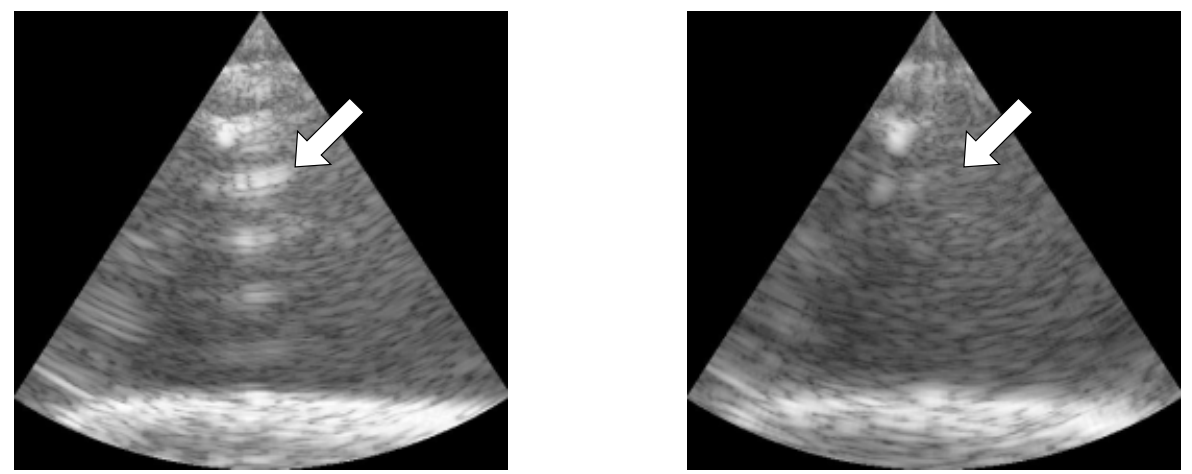

Fig. 6. One frame from the phantom sequence, before (left) and after (right) filtering. The reverberation artifact marked as noise by the operator is pointed out.

With an increased number of dimensions, the degrees of freedom of the filter is increased. One dimensional global filters will not be able to reconstruct a signal where noise has a spatial extent. Using a 2D filter the correlation between images adjacent in time is not exploited. In this study, where a volume consisting of several beams in a time-sequence is to be reconstructed, movements from e.g. respiration gives the reverberation artifact a periodic pattern, which can be better handled with a three dimensional filter as this account for a much more selective description of the artifact.

\section{Acknowledgments}

This work was supported by Amersham Health and performed within the NIMED competence center within Linköping University, Sweden. We are grateful to Birgitta Janerot Sjöberg, MD, PhD, for providing us with the in vivo ultrasound data sequence and valuable clinical support. 

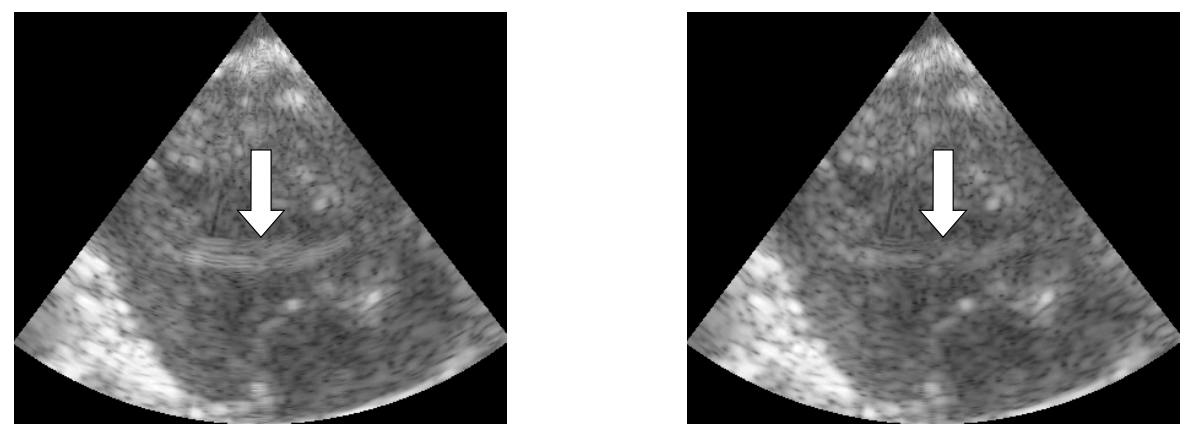

Fig. 7. One frame from the in vivo sequence, before (left) and after (right) filtering. The reverberation artifact is pointed out.

\section{References}

1. M. A. Averkiou. Tissue harmonic ultrasonic imaging. Comptes Rendus de l'Académie des Sciences - Series IV - Physics, Volume 2, Issue 8:1139-1151, October 2001.

2. N. Eriksson Bylund, M. Andersson, and H. Knutsson. Wide range frequency estimation in ultrasound images. In Proceedings of the SSAB Symposium on Image Analysis, March 2001.

3. F. Dong, E.L. Madsen, M.C. MacDonald, and J.A. Zagzebski. Nonlinearity parameter for tissue-mimicking materials. Ultrasound in Medicine $\mathscr{E}$ Biology, Vol. 25, Issue 5:831-838, 1999.

4. G. H. Granlund and H. Knutsson. Signal Processing for Computer Vision. Kluwer Academic Publishers, 1995. ISBN 0-7923-9530-1.

5. J. Kirkhorn. Introduction to IQ-demodulation of RF-data. Technical report, IFBT, NTNU, 1999.

6. M. Nickel. Modeling and reduction of reverberation noise in medical ultrasound images. PhD thesis, Norwegian university of science and technology, 1997.

7. J. U. Quistgaard. Ultrasonic image formation: implications for the image processing practitioner. In Image Processing, 1994. Proceedings. ICIP-94., IEEE International Conference.

8. A. Rosenfeld and A. C. Kak. Digital Picture Processing. Academic Press, Inc., second edition, 1982. ISBN 0-12-597301-2. 